\title{
Long-term Timing of 374 Pulsars
}

\author{
G. Hobbs
}

Australia Telescope National Facility - CSIRO, P.O. Box 76, Epping NSW 1710, Australia

\author{
A. Lyne, M. Kramer \\ Jodrell Bank Observatory, University of Manchester, Macclesfield, \\ Cheshire SK11 9DL, UK
}

\begin{abstract}
We have analyzed the arrival times for 374 pulsars that have been observed for more than six years using the 76-m Lovell telescope at Jodrell Bank Observatory. Here we present a qualitative analysis of structures seen in the timing residuals.
\end{abstract}

\section{Introduction}

More than 500 pulsars are being regularly observed using the $76-\mathrm{m}$ Lovell radio telescope at Jodrell Bank Observatory at frequencies between 408 and $1630 \mathrm{MHz}$. This archive contains more than 5600 years of pulsar rotational history which we supplement, for 18 pulsars, with early observations from the Jet Propulsion Laboratory (Downs \& Reichley 1983) to provide individual data spans of up to 34 years. In a series of three papers we plan to carry out a full analysis of the data for more than 350 pulsars that have been observed for longer than six years. The first paper provides accurate timing solutions including proper motion measurements. In the second paper we will use these proper motion values to improve our understanding of pulsar velocities. In the third paper we will discuss the remnant structures in the timing residuals after fitting a timing solution for rotational frequency and its first derivative. Some qualitative results, which will be discussed in more detail in the third paper, are highlighted here.

\section{Structure in the Timing Residuals}

Many different structures are observed in the timing residuals, including cubic features, cusps, flat residuals, pseudo-sinusoids and more irregular features. Cusps (corresponding to local maxima) indicate the pulsar undergoing a glitch. Pseudo-sinusoidal variations in the timing residuals may be caused by the pulsar precessing or from unmodeled binary companions.

The amplitudes of the observed cubic structures are many orders of magnitude greater than those predicted from magnetic dipole radiation; measured "braking indices" range between $\pm 3 \times 10^{8}$. 

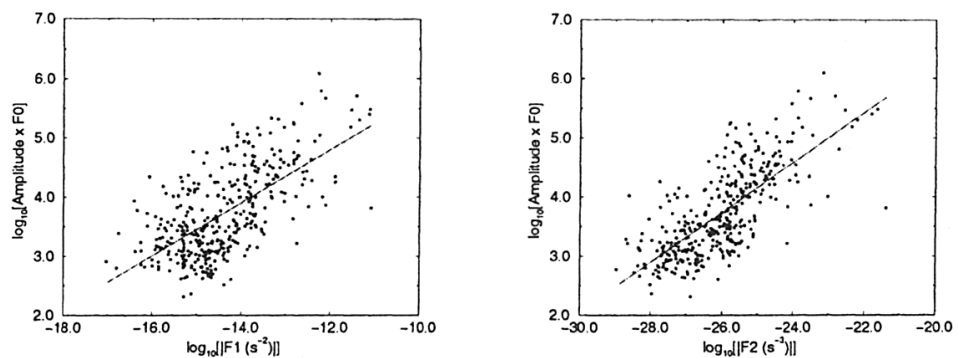

Figure 1. The rms amplitude of the timing noise plotted against rotational frequency first- (left panel) and second- (right panel) derivatives.

The structures observed in the majority of the timing residuals are not timeresolved. For example, about $30 \%$ are dominated by a large-scale cubic feature and a further $40 \%$ currently have no clearly defined features. However, we have a sample of 29 pulsars for which it is possible to study individual features in detail. We find that the distribution of the residuals around the mean value has no general asymmetry (with 15 having negative skew and 14 positive). We do find that $70 \%$ have, on average, sharper (larger second derivative) local maxima than local minima. No explanation is given for this effect at the present time.

The time corresponding to the first local minima in the auto-correlation function of the timing residuals provides an estimate of the time-scale of the structures present. However, we see no correlation of this parameter with the observed or derived pulsar parameters. We do find strong correlations between the amplitude of the timing noise and the first and second rotational frequency derivatives (Fig. 1).

Our new sample of timing residuals that span up to $\sim 30$ years will allow the first large scale analysis of timing noise to be carried out. Already it is clear that many of the structures observed in the timing residuals cannot have been caused by a simple "noise" process.

Acknowledgments. Many people have been involved in timing pulsars at Jodrell Bank. We thank, in particular, Christine Jordan for her work in developing much of the software used in the analysis of the observations.

\section{References}

Downs, G. S., \& Reichley, P. E. 1983, ApJS, 53, 169 